\title{
An uncoded BER comparison between DFE-SCCP and OFDM using a convex analysis framework
}

\author{
Amanda de Paula, Cristiano Panazio \\ Escola Politécnica, University of São Paulo, Brazil \\ Email: \{amanda,cpanazio\}@lcs.poli.usp.br
}

\begin{abstract}
This article aims to establish a theoretical performance comparison between the Single Carrier with Cyclic Prefix (SCCP) equalized with a Decision Feedback Equalizer (DFE) and the Orthogonal Frequency Division Multiplexing (OFDM) systems. We consider an uncoded scenario and we derive analytical relations between these systems's bit error rate (BER). The comparison is accomplished for different modulation schemes.
\end{abstract}

\section{INTRODUCTION}

Orthogonal frequency division multiplexing (OFDM) has become a popular transmission technique in part due to its low complexity equalization scheme based on the use of a cyclic prefix (CP) and a frequency domain one-tap equalizer. On the other hand, a single-carrier (SC) modulation scheme with the same CP solution can also use the same low complexity frequency domain one-tap equalizer structure, giving rise to the SCCP scheme [1]-[3].

Even though the OFDM presents clear advantages regarding the implementation aspect, since the received signal is free from intersymbol interference (ISI), if we assume that no channel information is known at the transmitter side, the OFDM highly depends on the coding scheme to properly exploit the frequency domain diversity [4], [5]. In contrast, the SCCP can be an interesting alternative to OFDM, since it naturally exploit the channel frequency diversity spreading the information throughout the used spectrum. Several papers compare these two transmission schemes regarding the biterror rate (BER) performance [1], [2], [4], [6]. Most of them are limited to quadrature phase-shift keying (QPSK) modulations, with a few exceptions that use M-ary quadrature amplitude modulation (QAM). Specifically, in [6], the authors prove that a SCCP with a linear equalizer (LE-SCCP) outperforms the OFDM, when the symbols are modulated with QPSK for uncoded systems, given that a minimum mean square error (MMSE) equalizer is employed. Also in [6], it is shown that this conclusion can not be generalized to higher order modulation schemes. Still, [6] does not analyze the BER of the decision feedback equalizer (DFE), which is known to present a superior performance when compared to the LESCCP [7].

Hence, a natural question that arises is if this improvement in performance provided by the DFE is enough to overcome the OFDM in any case where higher order modulation schemes are used. In this article, we try to answer this question through

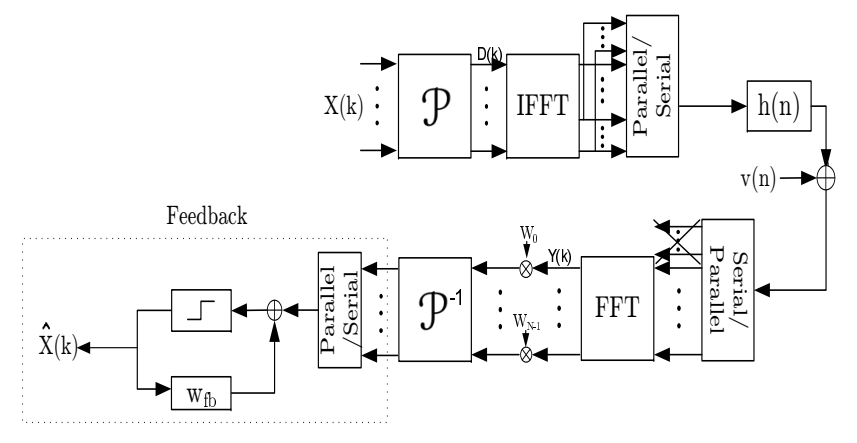

Fig. 1. System model

an analytical comparison between the DFE-SCCP and the OFDM systems in the uncoded scenario.

The rest of this paper is organized as follows. The system model is presented in Section II. In Section III, we present the performance comparison in terms of BER between the OFDM and the DFE systems by the means of a convex analysis framework. In Section IV, we present an asymptotic behavior analysis and, finally, in Section V, conclusions are stated.

\section{System Model}

The similarities between the OFDM and SCCP allow us to describe both modulation schemes in a unified system model [6] depicted in Fig. 1.

The two modulation schemes are distinguished by the linear precoding matrix $\mathcal{P}$. In the OFDM case, the transmitted symbols are obtained from the inverse fast Fourier transform (IFFT) of the data vector $\mathbf{X}$, thus the data vector is not preprocessed and the transformation $\mathcal{P}$ is replaced by the identity matrix. On the other hand, in the SCCP scheme the symbol vector itself is transmitted. In order to accomplish that, the precoding matrix $\mathcal{P}$ must be replaced by the Fourier matrix. The CP insertion allows us to equalize the received signal in the frequency domain with a simple one-tap equalizer.

If the SCCP system is equalized with a DFE, a feedback filter should be appended in the receiver. As illustrated in Fig. 1 , the feedforward filtering is accomplished in the frequency domain and the feedback filtering is implemented in the time domain. The feedback and feedforward filters coefficients can be calculated using the MMSE criterion as shown in [3] and the number of coefficients of the feedback filter is equal to the channel length minus one [8]. The DFE implementation with $\mathrm{CP}$ brings a problem in the initialization of the feedback filter, 
since it requires the access to the last symbols of the block, which have not yet been processed. An alternative to overcome this problem is to implement the DFE with the unique word (UW) technique [9]. With such approach, we can have the same rate or efficiency than the one obtained with the $\mathrm{CP}$ implementation, but a larger fast Fourier transform (FFT) is required. If the FFT length is the same for both approaches, the rates are different. However, if the block is large compared to the $\mathrm{CP}$ or UW lengths, the difference between both techniques may not be significative. Hence, in order to simplify the analysis, we will consider the DFE-SCCP and assume that the last symbols of the block are known and they are used to initialize the feedback filter. We also assume perfect feedback decisions to make the mathematical analysis more feasible.

Concerning the OFDM transmission scheme, the received symbols are free from intersymbol interference. In such a case, the frequency domain equalizer coefficients $\left(W_{k}\right)$ perform a simple magnitude and phase correction of the received symbol.

\section{Performance Comparison}

In this section we compare the BER expressions for both systems. As discussed in [7], the BER for M-QAM modulations can be approximated as:

$$
\mathrm{BER}=\alpha Q(\sqrt{\beta \mathrm{SNR}})
$$

where SNR is the signal-to-noise ratio in the equalizer output, $Q(x)$ is the complementary error function and the constants $\alpha$ and $\beta$ are given by:

$$
\alpha=\frac{4}{\log _{2}(M)}\left(1-\frac{1}{\sqrt{M}}\right) \quad \beta=\frac{3}{M-1}
$$

The approximation in eq. (1) is valid when Gray mapping is employed and for mild and high SNR values.

In the OFDM context, the received signal in the $k^{t h}$ subcarrier is expressed as:

$$
Y_{k}=H_{k} X_{k}+\eta_{k}
$$

where $H_{k}$ is the channel frequency response in the subcarrier, $X_{k}$ is the transmitted symbol and $\eta_{k}$ is the white Gaussian noise with zero mean and variance $\sigma_{\eta}^{2}$.

Thus, the SNR is different for each subcarrier and is given by:

$$
\mathrm{SNR}_{\mathrm{OFDM}, \mathrm{k}}=\gamma\left|H_{k}\right|^{2}
$$

where $\gamma=\frac{\sigma_{X}^{2}}{\sigma_{\eta}^{2}}$.

Therefore, the mean BER in an OFDM system with $N$ subcarriers is the following:

$$
\mathrm{BER}_{\mathrm{OFDM}}=\frac{1}{N} \sum_{k=0}^{N-1} \alpha Q\left(\sqrt{\beta \gamma\left|H_{k}\right|^{2}}\right)
$$

Conversely, for the SCCP, the symbol is spread among all the subcarriers. If the signal is equalized with a DFE and if we do not take in account the error propagation effect, the SNR is given by [10]:

$$
\mathrm{SNR}_{\mathrm{DFE}}=\exp \left\{\frac{1}{N} \sum_{k=0}^{N-1} \log \left(1+\gamma\left|H_{k}\right|^{2}\right)\right\}-1
$$

This expression can also be rewritten in terms of the geometric mean operator:

$$
\operatorname{SNR}_{\mathrm{DFE}}=\text { geomean }\left\{1+\gamma|\mathbf{H}|^{2}\right\}-1
$$

One could also equalize the SCCP signal with a linear equalizer. In such a case, it can be shown [11], that the expression of the SNR in the equalizer output is very similar to eq. (7), with the difference that it is given in terms of the harmonic mean instead of the geometric mean. It is known that the geometric mean is equal or greater than the harmonic mean. Therefore, we can conclude that $\mathrm{SNR}_{\mathrm{DFE}} \geq \mathrm{SNR}_{\mathrm{LE}}$. In an additive white Gaussian noise (AWGN) channel, all subcarriers experience the same SNR. In such a case, the harmonic and geometric means are equal and the equality between the SNR holds. As the function $Q(x)$ is strictly decreasing, eq. (1) guarantees that $\mathrm{BER}_{\mathrm{DFE}} \leq \mathrm{BER}_{\mathrm{LE}}$.

Our intention is to compare BER $\mathrm{OFDM}_{\mathrm{D}}$ given by eq. (5) and $\mathrm{BER}_{\mathrm{DFE}}$ given by:

$\mathrm{BER}_{\mathrm{DFE}}=\alpha Q\left(\sqrt{\beta\left[\exp \left\{\frac{1}{N} \sum_{k=0}^{N-1} \log \left(1+\gamma\left|H_{k}\right|^{2}\right)\right\}-1\right]}\right)$

In order to do so, we define the following auxiliary functions:

$$
\begin{gathered}
\psi(x)=\alpha Q(\sqrt{\beta x}) \\
\phi(x)=\log (x+1) \\
t(x)=\psi\left(\phi^{-1}(x)\right)=\alpha Q(\sqrt{\beta(\exp (x)-1)})
\end{gathered}
$$

The eqs. (5) and (8) can be rewritten as:

$$
\begin{gathered}
\mathrm{BER}_{\mathrm{OFDM}}=\frac{1}{N} \sum_{k=0}^{N-1} \psi\left(\gamma\left|H_{k}\right|^{2}\right)=\frac{1}{N} \sum_{k=0}^{N-1} t\left(\phi\left(\gamma\left|H_{k}\right|^{2}\right)\right) \\
\mathrm{BER}_{\mathrm{DFE}}=\psi\left(\phi^{-1}\left(\frac{1}{N} \sum_{k=0}^{N-1} \phi\left(\gamma\left|H_{k}\right|^{2}\right)\right)\right)=t\left(\frac{1}{N} \sum_{k=0}^{N-1} \phi\left(\gamma\left|H_{k}\right|^{2}\right)\right)
\end{gathered}
$$

The equations above allow us to compare the BER expressions invoking concavity properties. In order to explore the concavity properties of $t(x)$, we must analyze its second derivative:

$$
\frac{d^{2}}{d x^{2}} t(x)=\underbrace{\left(1-\frac{\beta e^{x}}{2}\left(1+\frac{1}{\beta\left(e^{x}-1\right)}\right)\right)}_{a(x)} \frac{d}{d x} t(x)
$$

The function $t(x)$ is the composition of a strictly decreasing function with a strictly increasing function. Therefore, $t(x)$ is strictly decreasing and its first derivative $\frac{d}{d x} t(x)$ is negative on the whole domain interval.

By definition, a function is convex if and only if its second derivative is non-negative. Thus, a necessary and sufficient 


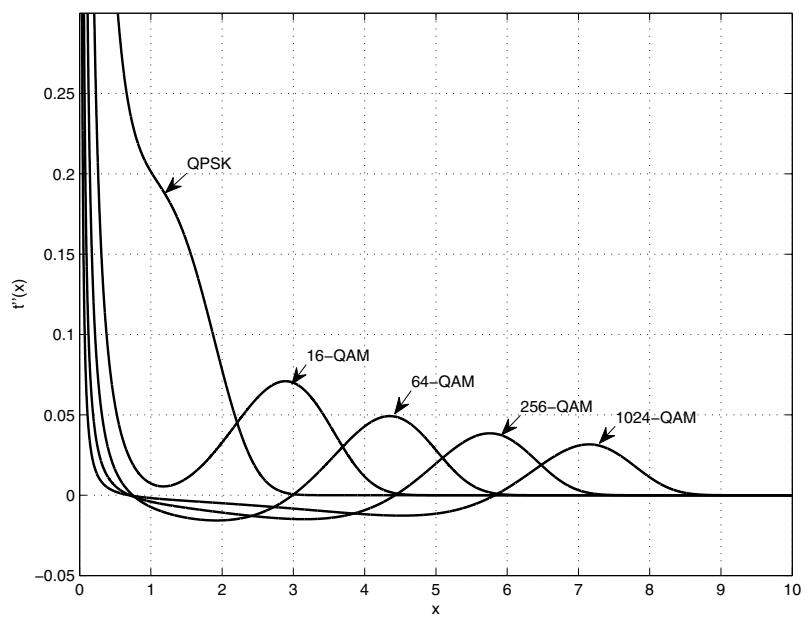

Fig. 2. Function $\mathrm{t}(\mathrm{x})$ - second derivative

condition to $t(x)$ be convex is that the function $a(x)$ is nonpositive. It can be shown that the symbol mapping QPSK, as well as the 16-QAM, leads to $\beta$ values that guarantee that the function $a(x)$ is non-positive on the whole domain interval. If the function $t(x)$ is convex, i.e. $\frac{d^{2}}{d x^{2}} t(x) \geq 0$, the Jensen's inequality [12] guarantees that:

$$
t\left(\frac{1}{N} \sum_{k=0}^{N-1} \phi\left(\gamma\left|H_{k}\right|^{2}\right)\right) \leq \frac{1}{N} \sum_{k=0}^{N-1} t\left(\phi\left(\gamma\left|H_{k}\right|^{2}\right)\right)
$$

Therefore, for these modulations we conclude that:

$$
\mathrm{BER}_{\mathrm{DFE}} \leq \mathrm{BER}_{\mathrm{OFDM}}
$$

Concerning QPSK and 16-QAM modulations, the above inequality holds independently of the channel configuration. For higher order modulation schemes, the function $t(x)$ presents both concavity and convexity intervals, as can be inferred from Fig. 2.

As an example, we consider transmission over the unitary norm channel with the following transfer function:

$$
H(z)=\prod_{k=1}^{4}\left(1-\rho_{k} \exp \left(j \delta_{k}\right) z^{-1}\right)
$$

where $\delta_{1,3}=\frac{\pi}{4}, \delta_{2,4}=-\frac{\pi}{4}, \rho_{1,2}=\frac{1}{2}, \rho_{3,4}=2$.

The Fig. 3 shows the theoretical BER considering QPSK, 16-QAM and 256-QAM modulations. Concerning QPSK and 16-QAM modulations, one can notice that the DFE-SCCP surpasses the OFDM for any SNR condition. When the 256QAM modulation is employed, on the other hand, the OFDM slightly surpass the DFE-SCCP for some SNR values.

\section{Asymptotic PERFormance COMPARISON}

In the previous section, we showed that the OFDM system can surpass the SCCP system for some modulation schemes and it was also shown that this situation occurs in a low SNR scenario. In this section, we consider an asymptotic scenario where $\gamma \gg 1$, i.e., BER $\rightarrow 0$.

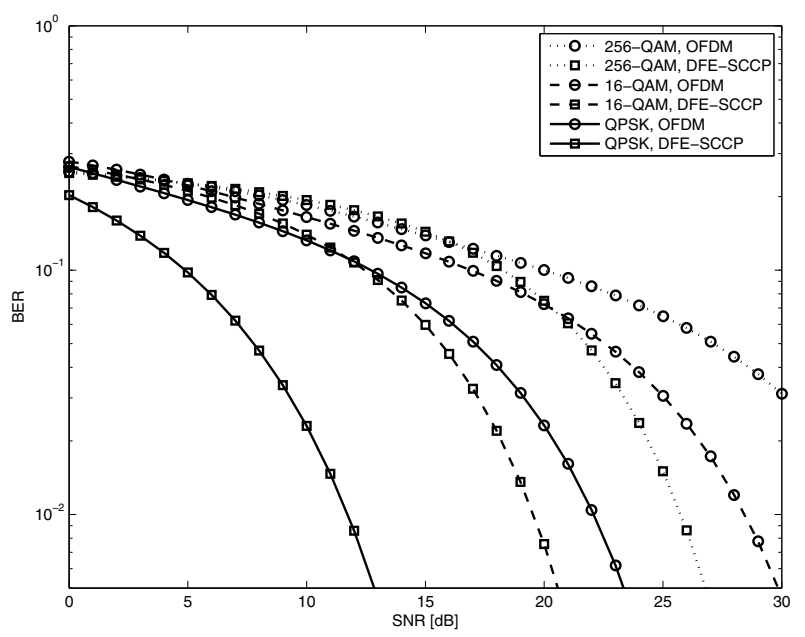

Fig. 3. Performance comparison

In order to compare the system in a low BER condition, we derive the performance gap $\Delta=\frac{\gamma_{2}}{\gamma_{1}}$, where $\gamma_{1}$ and $\gamma_{2}$ are such that:

$$
\operatorname{BER}_{\mathrm{SCCP}}\left(\gamma_{1}\right)=\operatorname{BER}_{\mathrm{OFDM}}\left(\gamma_{2}\right)
$$

in the particular case that $\gamma_{1}, \gamma_{2} \rightarrow \infty$.

In this situation, we can invoke the Chernoff bound and approximate the error function $Q(x)$ as:

$$
Q(x) \approx \frac{1}{2} \exp \left(-\frac{x^{2}}{2}\right)
$$

This approximation leads to the following BER expression for the DFE-SCCP system:

$$
\operatorname{BER}_{\mathrm{DFE}}\left(\gamma_{1}\right)=\frac{\alpha}{2} \exp \left(-\beta \frac{\operatorname{SNR}_{\mathrm{DFE}}\left(\gamma_{1}\right)}{2}\right)
$$

For BER $\rightarrow 0$, i.e., $\gamma_{1} \rightarrow \infty$, the $\mathrm{SNR}_{\mathrm{DFE}}$ can be approximated as:

$\operatorname{SNR} R_{\text {DFE }}\left(\gamma_{1}\right)=$ geomean $\left(\gamma_{1}|\mathbf{H}|^{2}\right)=\gamma_{1}$ geomean $\left(|\mathbf{H}|^{2}\right)$

By contrast, for the OFDM system, the BER is given by:

$$
\operatorname{BER}_{\text {OFDM }}\left(\gamma_{2}\right)=\frac{1}{N} \sum_{k=0}^{N-1} \frac{\alpha}{2} \exp \left(-\beta \frac{\gamma_{2}\left|H_{k}\right|^{2}}{2}\right)
$$

Moreover, we may define $\Gamma_{\text {OFDM }}$ as:

$\Gamma_{\text {OFDM }}\left(\gamma_{2}\right)=-\frac{2}{\beta} \log \left(\frac{1}{N} \sum_{k=0}^{N-1} \exp \left(\frac{-\beta \gamma_{2}\left|H_{k}\right|^{2}}{2}\right)\right)$

and than:

$$
\operatorname{BER}_{\mathrm{OFDM}}\left(\gamma_{2}\right)=\frac{\alpha}{2} \exp \left(-\beta \frac{\Gamma_{\mathrm{OFDM}}\left(\gamma_{2}\right)}{2}\right)
$$

Since the exponential is an injective function, from eqs. (20) and (24), it can be stated that:

$\operatorname{BER}_{\text {DFE }}\left(\gamma_{1}\right)=\operatorname{BER}_{\mathrm{OFDM}}\left(\gamma_{2}\right) \leftrightarrow \operatorname{SNR}_{\mathrm{DFE}}\left(\gamma_{1}\right)=\Gamma_{\mathrm{OFDM}}\left(\gamma_{2}\right)$ 


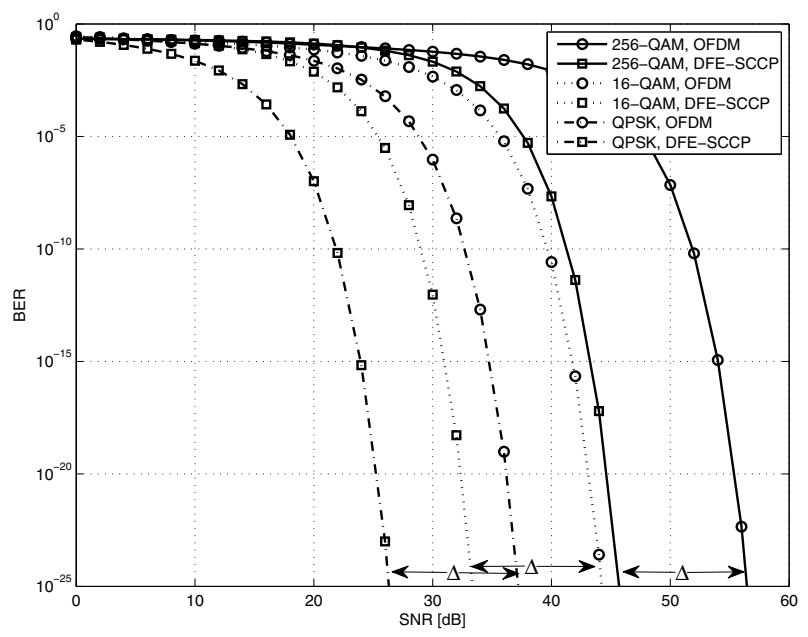

Fig. 4. Performance comparison for asymptotic behavior BER scenario

For high SNR values, the following approximation is valid:

$$
-\frac{2}{\beta} \log \left(\frac{1}{N} \sum_{k=0}^{N-1} \exp \left(\frac{-\beta \gamma_{2}\left|H_{k}\right|^{2}}{2}\right)\right) \approx \gamma_{2}\left|H_{m i n}\right|^{2}
$$

where $\left|H_{\min }\right|^{2}$ is given by:

$$
\left|H_{\text {min }}\right|^{2}=\underset{k}{\operatorname{argmin}}\left|H_{k}\right|^{2}
$$

Furthermore, we determinate the gap $\Delta$ from eqs. (18), (21) and (26):

$$
\gamma_{2}\left|H_{\text {min }}\right|^{2}=\gamma_{1} \text { geomean }\left(|\mathbf{H}|^{2}\right)
$$

This is equivalent to state that:

$$
\Delta=\frac{\text { geomean }\left(|\mathbf{H}|^{2}\right)}{\left|H_{\min }\right|^{2}}
$$

At this point it is worthwhile to emphasize the following relation is valid [12]:

$$
\left|H_{\text {min }}\right|^{2} \leq \text { geomean }\left(|\mathbf{H}|^{2}\right)
$$

This relation allows us to state that the performance gap $\Delta$ is always greater than one.

Other important aspect that should be emphasized is that this performance gap does not depend on the modulation order.

The Fig. 4 shows a comparison between the theoretical BER of the two schemes in a high SNR scenario. This performance comparison was accomplished considering the channel described in eq. (17). For this channel, the performance gap $\Delta$ is equal to $\Delta=11.08(\mathrm{~dB})$, this value is obtained applying eq. (29) to the chosen channel. This performance gap is indicated in the Fig. 4 showing that this gap is, indeed, independent of the modulation order.

\section{CONCLUSION}

In this article, we have established a BER comparison between OFDM and DFE-SCCP techniques for an uncoded system with a general frequency selective channel for QPSK and QAM modulations. We have shown that DFE-SCCP outperforms OFDM for QPSK and 16-QAM modulations for any given channel. However, for higher modulation this is not true anymore. From 64-QAM, the OFDM may outperform the DFE-SCCP for higher BER values. In addition, we showed that for lower BER values, the DFE-SCCP outperforms the OFDM and derived the performance gap between these two schemes in this condition. This performance gap was shown to be independent of the modulation scheme.

\section{REFERENCES}

[1] D. Falconer, S. L. Ariyavisitakul, A. Benjamin-Seeyar, and B. Edison, "Frequency domain equalization for single-carrier broadband wireless systems," IEEE Communications Magazine, vol. 40, pp. 58-66, April 2002.

[2] H. Sari, G. Karam, and I. Jeanclaude, "Transmission techniques for digital terrestrial TV broadcasting," IEEE Communications Magazine, vol. 33, no. 2, pp. 100-109, Feb. 1995.

[3] N. Benvenuto and S. Tomasin, "On the comparison between OFDM and single carrier modulation with a DFE using a frequency-domain feedforward filter," IEEE Trans. On Communications, vol. 50, no. 6, June 2002.

[4] F. Pancaldi, G. Vitetta, R. Kalbasi, N. Al-Dhahir, M. Uysal, and H. Mheidat, "Single-carrier frequency domain equalization," IEEE Signal Processing Magazine, vol. 25, pp. 37-56, 2008.

[5] V. Aue, G. P. Fettweis, and R. Valenzuela, "A comparison of the performance of linearly equalized single carrier and coded OFDM over frequency selective fading channels using the random coding technique," in Proc. Int. Conf. Communications (ICC), Atlanta, 1998, vol. 2, pp. 753-757.

[6] Yuan-Pei Lin and See-May Phoong, "BER minimized OFDM systems with channel independent precoders," Signal Processing, IEEE Transactions on, vol. 51, no. 9, pp. 2369-2380, Sept. 2003.

[7] M. Salehi J. Proakis, Digital Communications, Mc Graw-Hill, 5 edition, 2008.

[8] R. Lopez-Valcarce, "Realizable linear and decision feedback equalizers: properties and connections," Signal Processing, IEEE Transactions on, vol. 52, no. 3, pp. 757-773, March 2004.

[9] H. Witschnig, T. Mayer, A. Springer, A. Koppler, L. Maurer, M. Huemer, and R. Weigel, "A different look on cyclic prefix for SC/FDE," in Personal, Indoor and Mobile Radio Communications, 2002. The 13th IEEE International Symposium on, 2002, vol. 2, pp. $824-828$ vol.2.

[10] J. Salz, "Optimum mean-square decision feedback equalization," Bell Syst. Tech. J., vol. 52, pp. 1341-1373, Oct. 1973.

[11] B. Devillers, J. Louveaux, and L. Vandendorpe, "About the diversity in cyclic prefixed single-carrier systems," Elsevier Physical Communication, vol. 1, pp. 266-276, Dec. 2008.

[12] G. Pólya G. H. Hardy, J. E. Littewood, Inequalities, Cambridge, 1952. 